Please do not remove this page

RMIT

UNIVERSITY

\title{
Novel concept of a single-mass adaptively controlled triaxial angular rate sensor
}

John, James; Vinay, Thurai

https://researchrepository.rmit.edu.au/esploro/outputs/9921860163101341/filesAndLinks?institution=61RMIT_INST\&index=null

John, J., \& Vinay, T. (2006). Novel concept of a single-mass adaptively controlled triaxial angular rate sensor. IEEE Sensors Journal, 6(3), 588-595. https://doi.org/10.1109/jsen.2006.874458

Published Version: https://doi.org/10.1109/jsen.2006.874458

Repository homepage: https://researchrepository.rmit.edu.au

(c) 2006 IEEE. Personal use of this material is permitted. However, permission to reprint/republish this material for advertising or promotional purposes or for creating new collective works for resale or redistribution to servers or lists, or to reuse any copyrighted component of this work in other works must be obtained from the IEEE.

Downloaded On 2023/04/26 23:20:15 +1000 


\title{
Novel Concept of a Single-Mass Adaptively Controlled Triaxial Angular Rate Sensor
}

\author{
James D. John, Member, IEEE, and Thurai Vinay, Member, IEEE
}

\begin{abstract}
This paper presents a novel concept for an adaptively controlled triaxial angular rate (AR) sensor device that is able to detect rotation in three orthogonal axes, using a single vibrating mass. Pedestrian navigation is presented as an example demonstrating the suitability of the proposed device to the requirements of emerging applications. The adaptive controller performs various functions. It updates estimates of all stiffness error, damping and input rotation parameters in real time, removing the need for any offline calibration stages. The parameter estimates are used in feedforward control to cancel out their otherwise erroneous effects, including zero-rate output. The controller also drives the mass along a controlled oscillation trajectory, removing the need for additional drive control. Finally, the output of the device is simply an estimate of input rotation, removing the need for additional demodulation normally used for vibratory AR sensors. To enable all unknown parameter estimates to converge to their true values, the necessary model trajectory is shown to be a three-dimensional Lissajous pattern. A modified trajectory algorithm is presented that aims to reduce errors due to discretization of the continuous time system. Simulation results are presented to verify the operation of the adaptive controller. A finite-element modal analysis of a preliminary structural design is presented. It shows a micro electro mechanical systems realizable design having modal shapes and frequencies suitable for implementing the presented adaptive controller.
\end{abstract}

Index Terms-Adaptive, analysis, angular rate, control, FEA, gyroscope, MEMS, modal, modeling, sensor, triaxial.

\section{INTRODUCTION}

$\mathbf{T}$ HE adoption of micro electro mechanical systems (MEMS) technology for fabricating angular rate (AR) sensors has allowed the fabrication of smaller, cheaper and much more energy efficient devices than conventional macro-sized devices [19]. This in turn has enabled and encouraged new applications to emerge. One example of a major emerging application for these devices is pedestrian navigation in mobile devices to enable location-based services. GPS has been the main technology proposed as a solution; however it uses a lot of power, does not work indoors and suffers from signal reflections in city environments where location-based services will be used most. A better solution is to have an occasional GPS

Manuscript received February 8, 2005; revised April 17, 2005. The associate editor coordinating the review of this paper and approving it for publication was Prof. Paul Regtien.

The authors are with the School of Electrical and Computer Engineering, RMIT University, City Campus, Melbourne 3001, Vic., Australia (e-mail: james.d.john@gmail.com; thurai.vinay@ rmit.edu.au).

Digital Object Identifier 10.1109/JSEN.2006.874458 location reading followed by dead reckoning from that location using an inertial measurement unit (IMU) [11]. Generally an IMU can resolve acceleration and AR in three orthogonal axes. Any error in IMU output will be accumulated in the dead reckoning calculation over time, so it is important that each device is not only small but also accurate to enable the period between required GPS fixes to be extended.

The aim of this work is to develop an AR sensing device that is able to resolve input AR into three orthogonal axes using a single vibrating mass. Providing a triaxial AR sensor on one chip in a small package has been identified as a major thrust in inertial sensor technology trends [3].

The use of a single mass to sense triaxial rotation promises to reduce footprint size, which increases production yield and subsequently decreases cost and should reduce energy consumption given only one mass is driven into vibration instead of three separate ones. These advantages suit the requirements of many emerging applications such as the pedestrian navigation described above that require triaxial AR sensing as part of their inertial measurement.

Another advantage of using a single vibrating mass is that it avoids mechanical interference. This is experienced when multiple vibrating masses are mounted together or share a substrate and is amplified when they share similar resonant frequencies [10].

A review of the literature has uncovered one proposed method of resolving triaxial AR using a single vibrating mass [10]. It involves driving the mass in a circular trajectory in the $x-y$ plane. The proposed device uses an existing triaxial accelerometer [5]. It consists of a single cubic mass with three pairs of orthogonal displacement sensors assembled around it. The mass is then excited using additional electromagnetic actuation in two axes. This device however is quite large for a MEMS device $(3 \mathrm{~mm} \times 3 \mathrm{~mm} \times 3 \mathrm{~mm}$, not including the electromagnetic actuation elements and electronics), requires costly assembly of the displacement sensors around the mass and is vulnerable to output errors caused by fabrication imperfections and time varying effects. These are disadvantages that the proposed adaptive controlled triaxial AR sensor can overcome.

The proposed device consists of a single suspended mass that is free to move in three dimensions, has actuation elements in three orthogonal axes and has sensing elements that are able to resolve the mass's displacement and velocity in each axis with respect to the device casing. The device concept is shown in Fig. 1. 


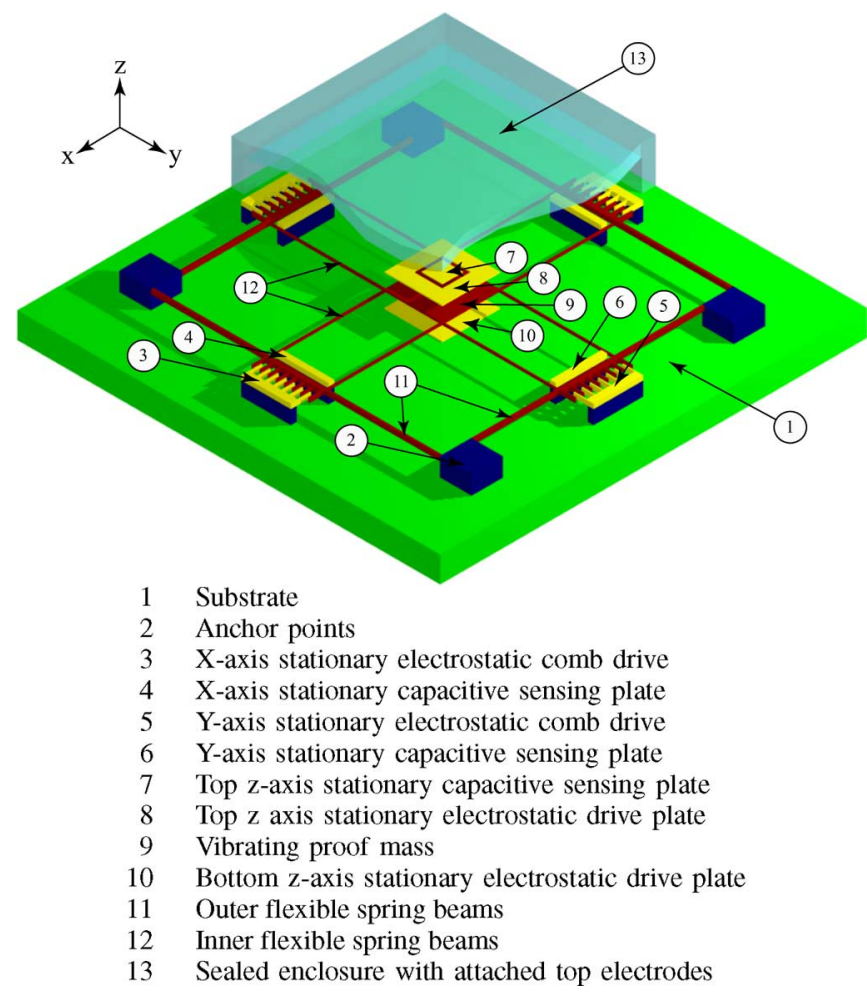

Fig. 1. Device structure concept drawing. (Color version available online at http://ieeexplore.ieee.org.)

\section{Single-MASS TRIAXIAL AR SENSOR}

\section{A. AR Sensor Dynamics Errors}

Much of the previous research into MEMS AR sensors has been aimed at developing new and improved fabrication techniques to create a device structure without imperfections [19]. Recent research work however is shifting the focus from trying to fabricate a perfect device to controlling an imperfect device's dynamics so it will behave like a perfect one [7], [8], [12], [13], [15], [17]. This can be achieved using feedforward control that compensates for structural imperfections and damping effects that would otherwise cause a zero rate output (ZRO) error [19]. Researchers have used control techniques that null the mass's angular momentum with respect to the device casing to reduce quadrature error and conserve system energy to cancel damping energy dissipation [13], [17]. Others have used adaptive control laws to update system parameter estimates that are then used in a feedforward control to null their erroneous effects and control the mass along an ideal reference trajectory [15]. They have managed to identify and compensate for cross damping errors, which are generally difficult to distinguish from angular rate and this approach has subsequently been adopted for this work.

To demonstrate how various error sources affect the dynamics of a triaxial AR sensor, nonideal dynamics of a real device are presented along with those of an ideal one.

It should be noted that all equations in this paper use nondimensionalised variables obtained by dividing each term in the real dynamic equation by the proof mass $m$ and a reference length $q_{0}$ (normally chosen as $1 \mu \mathrm{m}$ ). Each time derivative is also scaled by the reference frequency $\omega_{0}$ (normally chosen as the natural frequency in one of the axes). This technique of nondimensionalising follows that of [14] and [15].

Ideal triaxial AR sensor dynamics can be expressed in matrix form as

$$
\ddot{q}+K_{\text {ideal }} q=-2 \Omega \dot{q}
$$

where

$$
\begin{aligned}
K_{\text {ideal }} & =\left[\begin{array}{ccc}
\omega_{x}^{2} & 0 & 0 \\
0 & \omega_{y}^{2} & 0 \\
0 & 0 & \omega_{z}^{2}
\end{array}\right] \\
\Omega & =\left[\begin{array}{ccc}
0 & -\Omega_{z} & \Omega_{y} \\
\Omega_{z} & 0 & -\Omega_{x} \\
-\Omega_{y} & \Omega_{x} & 0
\end{array}\right] \\
q & =\left[\begin{array}{l}
x \\
y \\
z
\end{array}\right] .
\end{aligned}
$$

Equation (1) is of the form of a perfect oscillator. Given that there are no damping terms in the ideal case, there is no energy lost in the system, only a transfer of energy between axes through the Coriolis term $-2 \Omega \dot{q}$.

The principle stiffness terms $\omega_{x}^{2}, \omega_{y}^{2}$ and $\omega_{z}^{2}$ correspond to the squared nondimensional resonant frequencies of each axis.

A practical AR sensor has a similar dynamic form to (1)

$$
\ddot{q}+D \dot{q}+K q=-2 \Omega \dot{q}
$$

where

$$
\begin{aligned}
K & =\left[\begin{array}{lll}
k_{x x} & k_{x y} & k_{x z} \\
k_{x y} & k_{y y} & k_{y z} \\
k_{x z} & k_{y z} & k_{z z}
\end{array}\right] \\
D & =\left[\begin{array}{lll}
d_{x x} & d_{x y} & d_{x z} \\
d_{x y} & d_{y y} & d_{y z} \\
d_{x z} & d_{y z} & d_{z z}
\end{array}\right] .
\end{aligned}
$$

However, it has a nonzero damping matrix $D$. The stiffness matrix $K$ and damping matrix $D$ also have unwanted cross coupling terms between each of the three axes and the stiffness in the $\mathrm{x}, \mathrm{y}$, and $\mathrm{z}$ axes may not be as designed.

The stiffness matrix $K$ is always symmetric due to Maxwell's Reciprocity Theorem; similarly, $D$ is also symmetric [1]. The cross stiffness and cross damping terms can be thought of as causing a rotation of the respective stiffness and damping principle axes in three-dimensional (3-D) space from their ideal alignment with the $\mathrm{x}, \mathrm{y}$, and $\mathrm{z}$ axes.

There are many sources of error in a conventional AR sensor that contribute to the nonideal $K$ and $D$ matrices. Some of these effects can be time varying, such as those affected by temperature.

Random noise sources including thermal mechanical, sensor and circuitry noise will have a limiting effect on resolution, however these have not been included in this paper. 


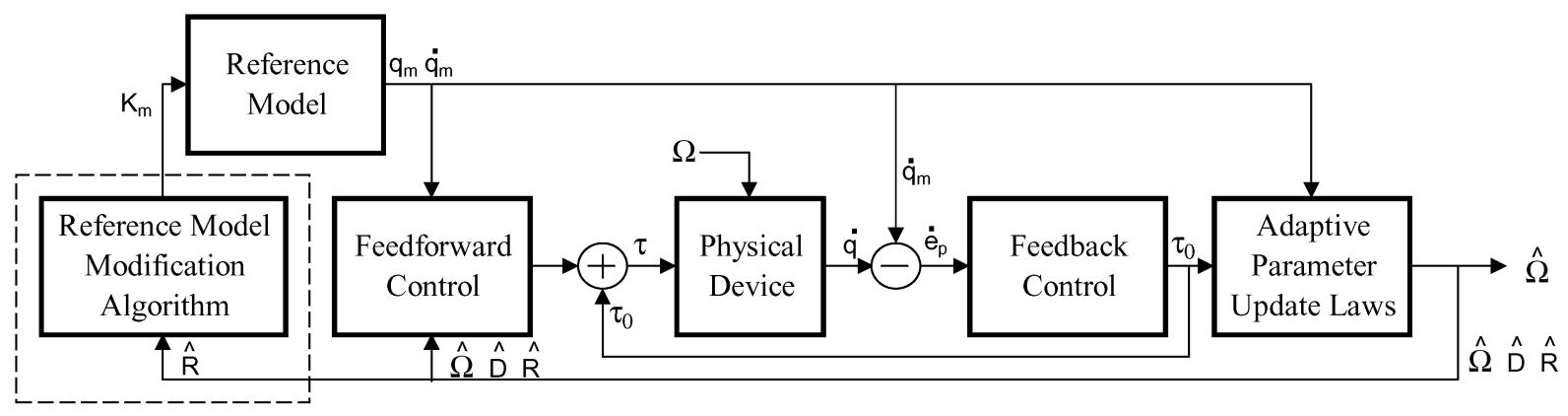

Fig. 2. Proposed adaptive control system diagram with the optional reference model modification stage.

\section{B. Adaptive Controller Development}

The operation of the adaptive controller for the triaxial AR sensor is outlined in Fig. 2. The fast changing displacement and velocity variables of the vibrating mass are compared to those of an ideal reference model trajectory, which will be developed in Section II-C. The error between the actual and model trajectories is then used by the adaptive update laws developed later in this section to update the estimates of all the unknown, slow changing parameters, namely the damping $D$, stiffness error $R$ and AR $\Omega$. These parameter estimates are then used in a feedforward control to compensate for their effects. The controller inherently drives the mass along a controlled oscillation trajectory, removing the need for additional drive control. Estimation and control stages have been kept separate so that the output of the device can simply be taken directly from the parameter estimates of the input rotations $\hat{\Omega}_{x}, \hat{\Omega}_{y}$ and $\hat{\Omega}_{z}$. This removes the need for additional demodulation stages normally required by AR sensors [4].

The following triaxial adaptive controller derivation has been adopted from that of the single axis system in [14], [15] and can be seen as an extension of the single axis AR sensor approach into a triaxial system. Notation and nondimensionalising conventions have also been kept consistent where possible. The triaxial system has redefined matrices and the optional reference model trajectory modification stage shown in the dotted area of Fig. 2, which is proposed in Section II-D to minimize the bias errors due to discretization of the continuous time system.

In the system block diagram of Fig. 2, the "Physical Device" block can be represented as

$$
\ddot{q}+D \dot{q}+K q=\tau-2 \Omega \dot{q}
$$

where $\tau$ is the sum of the feedforward and feedback control forces. It is subsequently defined in (10).

The reference model is similar to the dynamic equation of the ideal AR sensor in (1). However since the input rotation $\Omega$ is one of the unknown parameters, the Coriolis term is removed from the reference model and added to the feedforward control law $\tau$ so that it can be estimated and compensated in a similar manner to $K$ and $D$.

Therefore, the reference model becomes

$$
\ddot{q}_{m}+K_{m} q_{m}=0
$$

Here, $K_{m}$ is identical to $K_{\text {ideal }}$ in (3) with the constraint $\omega_{x} \neq \omega_{y} \neq \omega_{z}$ given in Section II-C. The $m$ subscripts denote parameters belonging to the reference model.

The estimated parameters are the input rotation estimate $\hat{\Omega}$, the estimate of the damping matrix of the real system $\hat{D}$ and the estimate of the error between the actual stiffness matrix and that of the reference model $\hat{R}=K-K_{m}$. These are continuously estimated and their effects compensated using feedforward control.

The control law $\tau$ is defined as

$$
\tau=\hat{D} \dot{q}_{m}+\hat{R} q_{m}+2 \hat{\Omega} \dot{q}_{m}+\tau_{0} .
$$

The first three terms form the feedforward control. There is an additional feedback control term $\tau_{0}$, defined as

$$
\tau_{0}=-\gamma \dot{e}_{p}
$$

with the positive definite gain matrix

$$
\gamma=\left[\begin{array}{ccc}
\gamma_{x} & 0 & 0 \\
0 & \gamma_{y} & 0 \\
0 & 0 & \gamma_{z}
\end{array}\right]
$$

and

$$
e_{p}=q-q_{m}
$$

The $\tau_{0}$ term is included to correct the mass's trajectory due to any inaccurate estimates and is subsequently used to correct the estimates in the adaptation laws (16)-(18). It also stabilizes the system given that the damping matrix $\mathrm{D}$ could be zero $(D \geq 0)$. This is explained further following (20) below.

Two good resources for the following Lyapunov-based adaptive controller design are [6] and [16].

Considering the positive definite Lyapunov function candidate $V$

$$
\begin{aligned}
V=\frac{1}{2}\left(\dot{e}_{p}^{T} \gamma \dot{e}_{p}+e_{p}^{T} \gamma K e_{p}\right. \\
\left.+\operatorname{tr}\left\{\gamma_{R}^{-1} \tilde{R} \tilde{R}^{T}+\gamma_{D}^{-1} \tilde{D} \tilde{D}^{T}+\gamma_{\Omega}^{-1} \tilde{\Omega} \tilde{\Omega}^{T}\right\}\right) .
\end{aligned}
$$

Note that for any practical device, the stiffness matrix $K$ in the Lyapunov function will be positive definite. 
Taking the time derivative of the above Lyapunov function (14) along the error trajectory gives

$$
\begin{aligned}
& \dot{V}=- \dot{e}_{p}^{T} \gamma(\gamma+D+2 \Omega) \dot{e}_{p} \\
&+\operatorname{tr}\left\{\tilde{R}\left(\gamma_{R}^{-1} \dot{\hat{R}}^{T}-\frac{1}{2} \dot{q}_{m} \tau_{0}^{T}-\frac{1}{2} \tau_{0} \dot{q}_{m}^{T}\right)\right. \\
&+\tilde{D}\left(\gamma_{D}^{-1} \dot{\hat{D}}^{T}-\frac{1}{2} q_{m} \tau_{0}^{T}-\frac{1}{2} \tau_{0} q_{m}^{T}\right) \\
&\left.+\tilde{\Omega}\left(\gamma_{\Omega}^{-1} \dot{\hat{\Omega}}^{T}-\dot{q}_{m} \tau_{0}^{T}+\tau_{0} \dot{q}_{m}^{T}\right)\right\}
\end{aligned}
$$

In order to make (15) negative semi-definite, the adaptation laws are chosen as

$$
\begin{aligned}
& \dot{\hat{R}}=\frac{1}{2} \gamma_{R}\left(\tau_{0} \dot{q}_{m}^{T}+\dot{q}_{m} \tau_{0}^{T}\right) \\
& \dot{\hat{D}}=\frac{1}{2} \gamma_{D}\left(\tau_{0} \dot{q}_{m}^{T}+\dot{q}_{m} \tau_{0}^{T}\right) \\
& \dot{\hat{\Omega}}=\gamma_{\Omega}\left(\tau_{0} \dot{q}_{m}^{T}-\dot{q}_{m} \tau_{0}^{T}\right) .
\end{aligned}
$$

This leaves

$$
\dot{V}=-\dot{e}_{p}^{T} \gamma(\gamma+D+2 \Omega) \dot{e}_{p}
$$

which, due to $\Omega$ being skew symmetric, leads to

$$
\dot{V}=-\dot{e}_{p}^{T} \gamma(\gamma+D) \dot{e}_{p}
$$

Given that $\gamma$ is positive definite and $D$ is positive semi-definite, then $\gamma+D$ will be positive definite and since $\dot{e}_{p}$ can be a zero vector, $\dot{V}$ is negative semi-definite and the Lyapunov stability criterion is satisfied. Since $D$ can be a zero matrix, $\gamma$ ensures that $\dot{V} \neq 0$, while $\dot{e}_{p} \neq 0$.

The outer parameter estimate loop is slow changing compared to the fast changing inner feedback control loop. In this respect the two loops can be considered as decoupled.

\section{Parameter Convergence}

Substituting the control law (10) into the system dynamics (8) gives

$$
\ddot{q}+(D+2 \Omega) \dot{q}+K q=(\hat{D}+2 \hat{\Omega}) \dot{q}_{m}+\hat{R} q_{m}+\tau_{0} .
$$

Expressing this in terms of the error vector $e_{p}$ gives the trajectory error dynamics

$$
\ddot{e}_{p}+(\gamma+D+2 \Omega) \dot{e}_{p}+K e_{p}=(\tilde{D}+2 \tilde{\Omega}) \dot{q}_{m}+\tilde{R} q_{m} .
$$

This shows that if the trajectory error and its derivatives approach zero $\ddot{e}_{p}, \dot{e}_{p}, e_{p} \rightarrow 0$ then given that $\dot{q}_{m}$ and $q_{m}$ are sinusoidal, $(\tilde{D}+2 \tilde{\Omega}) \rightarrow 0$ and $\tilde{R} \rightarrow 0$. Proof that $\ddot{e}_{p}, \dot{e}_{p}, e_{p} \rightarrow 0$ appears in [14] for the single axis case and can be shown to hold true for the triaxial case, with the redefined regressor $W$ and parameter error $\tilde{\theta}$ in (24).

We can describe the triaxial error dynamics in regressor form

$$
W^{T}\left(q_{m}, \dot{q}_{m}\right) \tilde{\theta}=\tilde{D} \dot{q}_{m}+\tilde{K} q_{m}+2 \tilde{\Omega} \dot{q}_{m}
$$

With the error vector $\tilde{\theta}$ and regressor $W\left(q_{m}, \dot{q}_{m}\right)$

$$
\tilde{\theta}=\left[\begin{array}{c}
r_{x x} \\
r_{y y} \\
r_{z z} \\
r_{x y} \\
r_{x z} \\
r_{y z} \\
d_{x x} \\
d_{y y} \\
d_{z z} \\
d_{x y} \\
d_{x z} \\
d_{y z} \\
\Omega_{x} \\
\Omega_{y} \\
\Omega_{z}
\end{array}\right], \quad W\left(q_{m}, \dot{q}_{m}\right)=\left[\begin{array}{ccc}
x_{m} & 0 & 0 \\
0 & y_{m} & 0 \\
0 & 0 & z_{m} \\
y_{m} & x_{m} & 0 \\
z_{m} & 0 & x_{m} \\
0 & z_{m} & y_{m} \\
\dot{x}_{m} & 0 & 0 \\
0 & \dot{y}_{m} & 0 \\
0 & 0 & \dot{z}_{m} \\
\dot{y}_{m} & \dot{x}_{m} & 0 \\
\dot{z}_{m} & 0 & \dot{x}_{m} \\
0 & \dot{z}_{m} & \dot{y}_{m} \\
0 & -2 \dot{z}_{m} & 2 \dot{y}_{m} \\
2 \dot{z}_{m} & 0 & -2 \dot{x}_{m} \\
-2 \dot{y}_{m} & 2 \dot{x}_{m} & 0
\end{array}\right]
$$

where each axis of the reference model is chosen to be sinusoidal

$$
\begin{array}{ll}
x_{m}=X_{0} \sin \left(\omega_{x} t\right), & \dot{x}_{m}=X_{0} \omega_{x} \cos \left(\omega_{x} t\right) \\
y_{m}=Y_{0} \sin \left(\omega_{y} t\right), & \dot{y}_{m}=Y_{0} \omega_{y} \cos \left(\omega_{y} t\right) \\
z_{m}=Z_{0} \sin \left(\omega_{z} t\right), & \dot{z}_{m}=Z_{0} \omega_{z} \cos \left(\omega_{z} t\right)
\end{array}
$$

where $X_{0}, Y_{0}$, and $Z_{0}$ are the amplitudes of vibration in the $x$, $y$, and $z$, axis respectively.

In order for all parameter estimates to converge to their true values, positive constants $\delta, \alpha_{1}$, and $\alpha_{2}$ must exist so that the persistence or excitation condition (26) is met [16]. This ensures the excitation gives enough information about the estimated parameters

$$
\alpha_{2} I \geq \int_{t}^{t+\delta} W\left(q_{m}, \dot{q}_{m}\right) W^{T}\left(q_{m}, \dot{q}_{m}\right) d \tau \geq \alpha_{1} I \quad \forall t \geq t_{0}
$$

where $I$ is an identity matrix.

As stated in [14], the persistence of excitation condition can always be met for the single $z$ axis AR sensor when the reference model's $x$ and $y$ axes resonant frequencies are unmatched $\left(\omega_{x} \neq \omega_{y}\right)$. Extending this unmatched resonant frequencies condition to the triaxial case gives $\omega_{x} \neq \omega_{y} \neq \omega_{z}$. This creates a 3-D Lissajous trajectory for the reference model, as can be seen in Fig. 3(a). Ideally, the persistence of excitation condition (26) with regressor $W^{T}\left(q_{m}, \dot{q}_{m}\right)$ (24) could be shown analytically to hold for all cases. This requires showing the positive definiteness of a $15 \times 15$ matrix. By inspection it is at least semi-definite, however proof of positive definiteness was found to be intractable by hand and too computationally intense using the symbolic software package Maple. Numerical substitutions using realistic case values indicate the persistence of excitation condition can be met when all modes are unmatched and has also been found analytically to always fail when all the resonant frequencies are matched. Although three sine waves appears to be insufficient excitation to allow the estimation of 15 parameters, additional higher frequencies are developed as a result of the cross coupling effects that provides additional information. Clearly, unmatched resonant frequencies are required for these higher frequencies to appear and integer multiples should also 


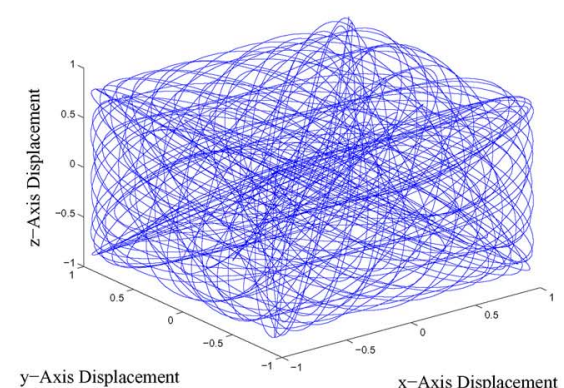

(a)

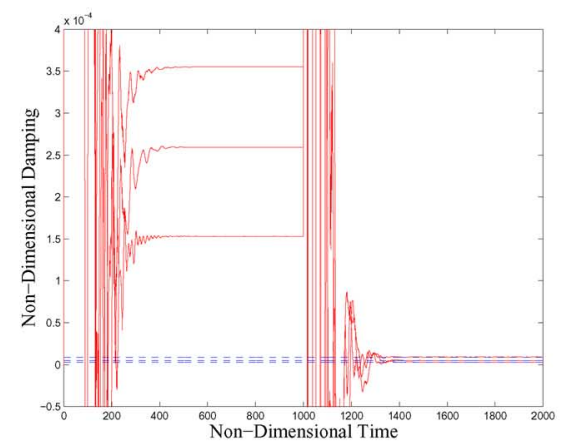

(d)

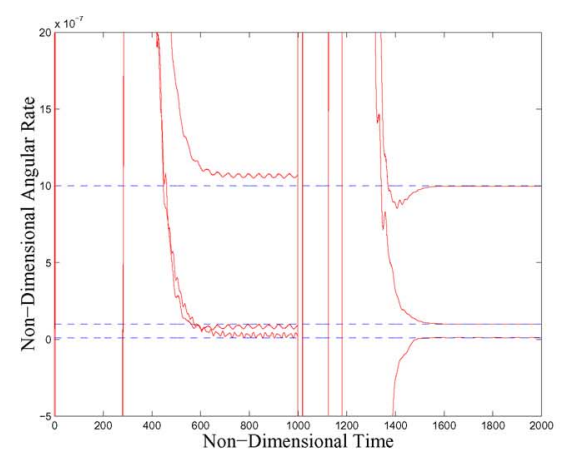

(b)

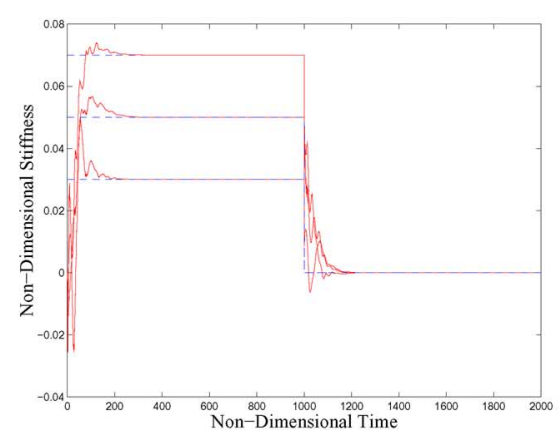

(e)

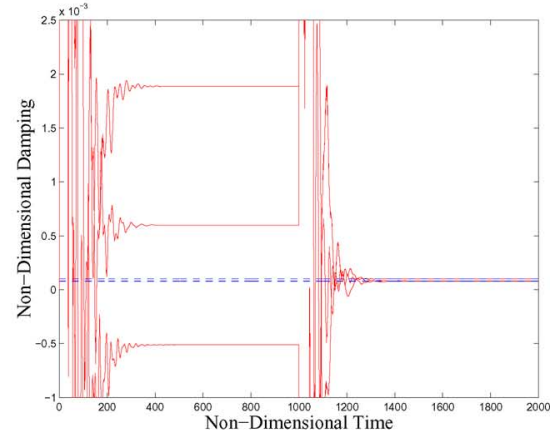

(c)

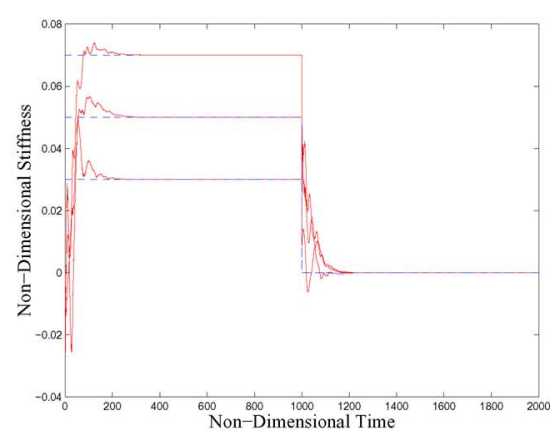

(f)

Fig. 3. Parameter estimates from the switched trajectory algorithm simulation. Actual values are indicated by the dashed line and are listed in Table I. (a) Reference model Lissajous trajectory prior to switching; (b) triaxial angular rate estimates $\hat{\Omega}$; (c) diagonal damping term estimates of $\hat{D}$; (d) cross damping term estimates of $\hat{D}$; (e) diagonal stiffness error term estimates of $\hat{R}$; (f) cross stiffness error term estimates of $\hat{R}$. (Color version available online at http://ieeexplore.ieee.org.)

be avoided. The adaptive controller was also implemented in MATLAB for a thorough simulation study, which supports the argument that the persistence of excitation condition is met for the triaxial case when $\omega_{x} \neq \omega_{y} \neq \omega_{z}$.

The simulations showed that the mass's trajectory converged to the reference model trajectory and the parameter estimates all converge to their true values. During the simulations, it was noted that there were bias errors in the parameter estimates, however these reduced as sample rate was increased. This is because the simulations are a digital implementation of the continuous time controller and as such introduce discretization errors. These errors were also encountered by [14], who proposed a dual rate discrete time controller to reduce the computation load. It proposed that adaptive parameter update laws (16)-(18) are updated using a slow sample rate given that the parameters being estimated are slow changing, while the control law (10) use a faster sample rate since it uses fast changing variables $q_{m}$ and $\dot{q}_{m}$.

The next section proposes a novel approach to reducing the size of the discretization bias error for the same sample rate.

\section{Modified Reference Model Trajectory Algorithm}

The bias errors were shown in [14] to be proportional to the greater of $\|R\|$ and $\omega_{x}\|D+2 \Omega\|$. Generally vibrating MEMS structures have high quality factors $(>1000)$ and therefore very low damping. The AR values are even smaller. Therefore even a small error in stiffness is likely to be the most significant parameter contributing to the discretization bias error for realistic
MEMS device. If this is the case the optional modified reference model trajectory algorithm is able to reduce $\|R\|$ by switching the reference model to match the estimated stiffness of the (possibly imperfect) physical device. To do this the stiffness error estimate $\hat{R}$ is added to the reference model

$$
\begin{aligned}
K_{m(\text { new })} & =K_{m(\text { old })}+\hat{R} \\
& =\left[\begin{array}{lll}
k_{m(x x)} & k_{m(x y)} & k_{m(x z)} \\
k_{m(x y)} & k_{m(y y)} & k_{m(y z)} \\
k_{m(x z)} & k_{m(y z)} & k_{m(z z)}
\end{array}\right] .
\end{aligned}
$$

This should only be done once the stiffness error estimate has converged sufficiently. The three principle stiffness axes of the reference model must, however, remain unmatched to maintain the 3-D Lissajous pattern, this could be done by setting nonoverlapping range limits on the reference model that can still accommodate a reasonable stiffness error. Since the reference model is redefined at a single point in time, it can be considered as independent from the operation of the adaptive parameter estimation and feedforward control. It can be seen as the system being reset with new initial conditions.

The reference model's 3-D Lissajous pattern will end up having similar principle axes to the principle stiffness axes of the physical device. It should be noted that the principle stiffness axes of the physical device may be misaligned from the $\mathrm{x}, \mathrm{y}$ and $\mathrm{z}$ axes due to fabrication imperfections.

When the switching occurs, all the parameter estimates deviate before converging closer to their true values. However the deviations are smaller if the reference model and physical device 
TABLE I

Simulation Results for the Adaptive Controller With the ReFEREnce Model Trajectory Switched to Match the Estimated StiffNess of THE Physical DeVICE at Time $=1000$ (All VAlues ARE IN Non-Dimensional Units)

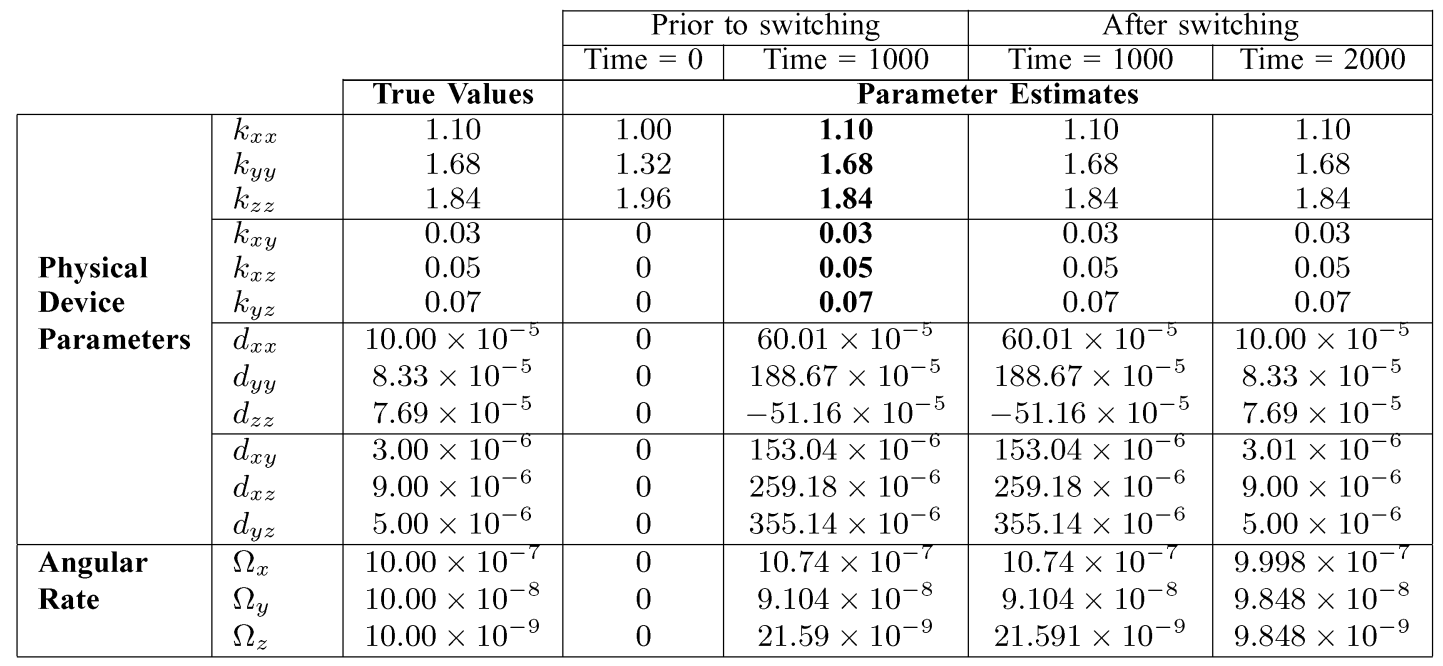

\begin{tabular}{|l|l|l|c|c|c|c|}
\cline { 4 - 6 } \multicolumn{2}{c|}{} & \multicolumn{4}{c|}{$\begin{array}{c}\text { Model trajectory switched at Time }=1000 \\
\text { to match the stiffness estimates }\end{array}$} \\
\hline \multirow{3}{*}{ Reference } & $k_{m(x x)}$ & NA & 1.00 & 1.00 & $\mathbf{1 . 1 0}$ & 1.10 \\
Model & $k_{m(y y)}$ & NA & 1.32 & 1.32 & $\mathbf{1 . 6 8}$ & 1.68 \\
& $k_{m(z z)}$ & NA & 1.96 & 1.96 & $\mathbf{1 . 8 4}$ & 1.84 \\
\cline { 2 - 7 } & $k_{m(x y)}$ & NA & 0 & 0 & $\mathbf{0 . 0 3}$ & 0.03 \\
& $k_{m(x z)}$ & NA & 0 & 0 & $\mathbf{0 . 0 5}$ & 0.05 \\
& $k_{m(y z)}$ & NA & 0 & 0 & $\mathbf{0 . 0 7}$ & 0.07 \\
\hline
\end{tabular}

are more closely matched. Therefore it is proposed that any realized device will store the last model trajectory in permanent memory. There could also be some decision logic that compares the $\hat{R}$ to $\omega_{x}\|D+2 \Omega\|$ to determine if the reference model trajectory requires switching.

Another possible implementation of this approach could use a reference model whose trajectory is slowly and continuously updated using the current stiffness error estimate. This approach should overcome the problem of the deviations seen in the switching algorithm, however it needs careful design so as not to interfere with the existing adaptive controller.

\section{E. Simulation Results}

For the purpose of this paper, results from the switched algorithm have been chosen to be presented. This is because the results of the switched algorithm clearly show the effect of modifying the reference trajectory. Also, the results prior to the trajectory being switched are representative of the unmodified system.

The triaxial adaptive control system with trajectory switching (Fig. 2) described in Section II-D has been simulated using the MATLAB package. A nonideal triaxial AR sensor structure was modeled by introducing errors in all the stiffness and damping matrices, including cross coupling terms between all three axes. The (nondimensional) values used for the presented simulation are summarized in Table I, as are the estimated values at two notable stages of the simulation namely at the time switching occurs $(T=1000)$ and at the end of the simulation $(T=$ 2000).
The simulation results can be split up into two sections: before and after the switching occurs. Before the switching occurs, all the estimates converge. There is, however, a bias in the estimates. This bias is due to discretization of the continuous time system and as such are reduced as the sample rate is increased. When the switching occurs, there are fluctuations in the estimates before they converge again but this time they converge much closer to their true values. This is due to the reduction in $\|R\|$ as discussed in Section II-D. The accuracy of the AR and damping estimates are dependent on the accuracy of the stiffness estimates when the switching occurs.

Since the adaptively controlled triaxial AR sensor is a novel device concept, it is important to show that the concept is realizable. This section has shown that the proposed adaptive controller has been simulated in software to verify that all unknown parameters including triaxial angular rate converge to their true values. Implementation of the system in software closely resembles that of a embedded controller on a real system. Section II-F addresses another challenge of implementing the proposed device which lies in designing a structure that is suitable for fabrication using standard MEMS techniques, namely being planar in design so as not to require assembly.

\section{F. Device Structure Design}

One imagines a single-mass triaxial AR sensor being identical in the three main axes, leading to a structure resembling a cube as was proposed by researchers [5], [10]. This approach however, does not translate well into the MEMS batch fabrication given that the design requires assembly steps due to its nonplanar design. The proposed adaptive controller also requires a 
slight mismatch in natural frequencies between each of the $\mathrm{x}, \mathrm{y}$, and $\mathrm{z}$ axes, leading to an inherently asymmetric design.

Ideally, the mass would remain parallel with respect to the device casing whilst vibrating laterally in the $\mathrm{x}, \mathrm{y}$, and $\mathrm{z}$ axes, i.e., a three degrees of freedom (DOF) system. However, given that they are inherently six DOF systems, every realizable device also has unwanted twisting modes. The aim thus becomes to design a six DOF system that, in its operating frequencies, behaves like a three DOF system. To achieve this the first three modes should be lateral motion in the $\mathrm{x}, \mathrm{y}$, and $\mathrm{z}$ axes, and the resonant frequency of the unwanted twisting modes should be sufficiently far away from the first three so as to not interfere with the operation of the device.

A preliminary device structure has been designed and modeled based on the structure shown in Fig. 1. In this process many considerations were made, including the suitability of the design for existing fabrication techniques, ensuring the displacements in the $\mathrm{x}, \mathrm{y}$, and $\mathrm{z}$ axes were independently measurable and that sensor element signals were free from unwanted rotational vibrations. As will be shown in Section II-F-1 the inherent six DOF system behaves like a three DOF system within the range of operating frequencies.

The structure design shown in Fig. 1 has been based on a symmetric single axis AR sensor [2]. However, the cross-section shape of the inner spring elements also allow the mass to vibrate in the $\mathrm{z}$-axis. It is proposed that the $\mathrm{x}$ and $\mathrm{y}$ axes motion will be sensed using differential capacitive combs and driven using electrostatic combs and that the z-axis motion will be sensed using capacitive plates and driven using electrostatic plates. Differential sensing will be used on the $\mathrm{x}$ and $\mathrm{y}$-axes to cancel out any unwanted "pull in" motion signals on the sensing elements due to displacement in a perpendicular axis.

A differential capacitive sensing approach has been presented in [9] and a similar circuit in [18], both are suitable for a triaxial device. Their single-ended approach simplifies the sensing circuitry and the physical connections of the capacitive electrodes. This is made possible by using the mass as a common electrode where a single supply Voltage is applied. The nominal capacitance of the sensing elements is expected to be in the $f F$ range; however, this will be dependent on the dimensions and number of electrodes.

It should be noted that the adaptive controller works independently of the sensing and actuation mechanisms used and that capacitive sensing and electrostatic actuation have been chosen for the preliminary design because of their suitability to silicon devices [3].

1) Structure Design and Modal Modeling: The proposed design has been modeled using the finite-element analysis (FEA) software ANSYS. Using the Block Lanczos mode extraction method the first six natural modal frequencies and their respective deformation shapes were found. After several design iterations, a structure was found whose first three modes were at similar frequencies and whose deformations were lateral in each of the $\mathrm{x}, \mathrm{y}$, and $\mathrm{z}$ axes. The following three modes are the unwanted twisting modes in the $\mathrm{x}, \mathrm{y}$, and $\mathrm{z}$ axes. This design was then slightly modified by shortening the length of the $\mathrm{x}$ axis outer spring element to create the slight mismatch in modal frequen-

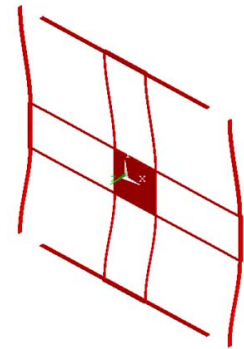

(a)

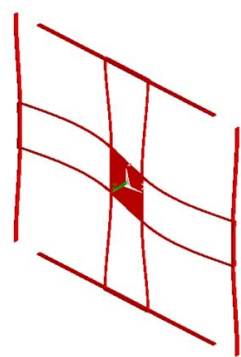

(d)

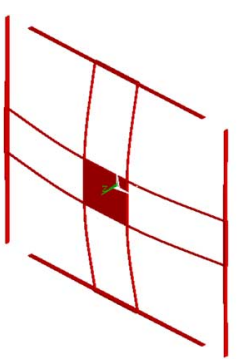

(b)

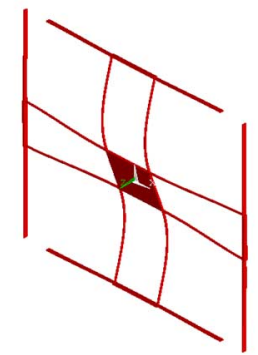

(e)

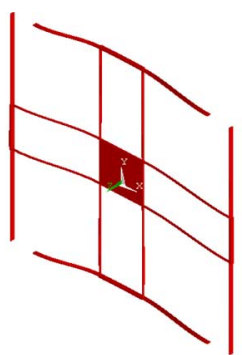

(c)

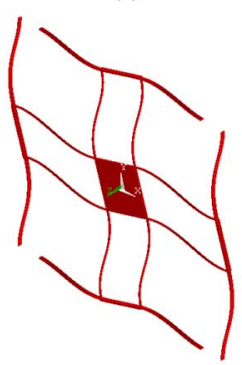

(f)
Fig. 4. First six modal shapes and respective frequencies of the proposed device structure as found by ANSYS modal analysis. (a) Mode 1, $35.7 \mathrm{kHz}$. (b) Mode 2, $41.1 \mathrm{kHz}$. (c) Mode 3, $49.7 \mathrm{kHz}$. (d) Mode 4, $169.0 \mathrm{kHz}$. (e) Mode 5, $186.4 \mathrm{kHz}$. (f) Mode 6, $193.9 \mathrm{kHz}$. (Color version available online at http://ieeexplore.ieee. org.)

cies between the $\mathrm{x}, \mathrm{y}$ and $\mathrm{z}$ axes needed by the adaptive controller described in Section II-C. The modal shapes of the first six resonant modes and their respective frequencies are shown in Fig. 4.

Initial modal analysis results have shown that a device made of silicon with feature dimensions achievable with standard silicon micromachining techniques can produce all three lateral vibration modes at similar but unmatched frequencies and the next mode, the first of the twisting modes, located at approximately three to four times the operating frequency region. Generally, micro structures have high quality factors with fast roll-off in their frequency response. Therefore, the effect of the rotational modes around the operation frequencies should be negligible. The ratios between the $\mathrm{x}, \mathrm{y}$, and $\mathrm{z}$ natural frequencies and the $\mathrm{x}$ axis frequency are $1,1.15$, and 1.40 as per the simulation initial estimate values used in Section II-E.

The size of the control forces $\tau$ are highly dependant on the difference between the reference model and the actual stiffness of the device. If they are exactly the same, only the damping dissipation and Coriolis forces need to be overcome, for the proposed device this is approximately $0.2 \mathrm{nN}$. However, if they are very different, larger control forces will be required conceivably up to the static deflection force which for the proposed device is approximately $20 \mu \mathrm{N}$. The voltages required to create these electrostatic forces vary depending on the comb drive dimensions however are in the range of one to tens of volts.

The proposed structure may also be designed with matched modes for implementation of the triaxial device operation proposed by [10] $p .78$. It has the advantages of being a planar structure suitable for existing fabrication processes and does not require any costly assembly steps. 


\section{DISCUSSION}

It can be seen from Table I that the AR estimates converge to their true values within approximately $\pm 2 e^{-9}$ which, with the modeled device resonant frequencies given in Section II-F, corresponds to $\pm 0.026 \mathrm{Deg} / \mathrm{s}$. Prior to switching the AR estimates converged within approximately $\pm 1 \mathrm{Deg} / \mathrm{s}$. This resolution limit is due to the discretization errors discussed in Sections II-B and II-D.

The effects of measurement errors including variance due to thermal mechanical and circuitry noise have not been considered in this work however it is anticipated that they will limit the ultimate achievable resolution of the AR estimates.

Detailed exploration of the convergence rate and transient behavior is also beyond the scope of this paper. However, it should be noted that they depend on the adaptation gains $\gamma, \gamma_{R}, \gamma_{D}$, and $\gamma_{\Omega}$, and ratios between the three mismatched natural frequencies $\omega_{x}, \omega_{y}$, and $\omega_{z}$.

The proposed triaxial adaptive scheme will require minimal energy during operation, because there is only one mass to be driven. Also, since the reference model will eventually be closely matched to the physical device, it will be driven at its natural resonant frequency in all axes and only damping dissipation forces will need to be overcome.

\section{CONCLUSION}

A novel single-mass adaptively controlled triaxial angular rate sensor has been presented. An existing adaptive control approach has been extended to a triaxial adaptive controller with necessary modifications.

In order for all parameter estimates to converge to their true values, it has been shown that the reference model needs to have unmatched resonant frequencies in its principle axes. The stiffness estimates can be used to match the reference model stiffness to the estimated device stiffness. This has been shown to reduce bias errors due to discretization of the continuous time system.

Simulation results have been presented that show the convergence of the AR and damping estimates to biased values before trajectory switching occurs and convergence closer to their true values after switching occurs.

A planar device structure suitable for MEMS fabrication has been modeled using FEA software and shown to have desirable lateral modal deformation shapes and frequencies in the $\mathrm{x}, \mathrm{y}$, and $\mathrm{z}$ axes. The resonant frequencies of the unwanted twisting modes are sufficiently far away from the operating frequency range so as not to interfere with the operation of the device.

\section{REFERENCES}

[1] S. Adhikari and J. Woodhouse, "Toward identification of a general model of damping," Proc. SPIE-Int. Soc. Opt. Eng., vol. 4062, no. I, pp. 377-383, 2000.

[2] S. E. Alper and T. Akin, "A symmetric surface micromachined gyroscope with decoupled oscillation modes," Sens. Actuators A: Phys., vol. 97-98, pp. 347-358, 2002.
[3] N. Barbour and G. Schmidt, "Inertial sensor technology trends," IEEE Sensors J., vol. 1, no. 4, pp. 332-339, Apr. 2001.

[4] J. John, C. Jakob, T. Vinay, and L. Q. , "Phase differential angular rate sensor-concept and analysis," IEEE Sensors J., vol. 4, no. 4, pp. 471-478, Apr. 2004.

[5] B. Kooi, W. Oltuis, and P. Bergveld, "Rate of turn sensing with a modified triaxial accelerometer," Eurosensors, 2000, Kopenhagen.

[6] M. Krstic, I. Kanellakopoulos, and P. Kokotovic, Nonlinear and Adaptive Control Design, ser. Adaptive and Learning Systems for Signal Processing Communications, and Control. New York: Wiley, 1995.

[7] R. Leland, "Adaptive tuning for vibrational gyroscopes," in Proc. 40th IEEE Conf. Decision and Control 2001, 2001, vol. 4, Theoretical or Mathematical, pp. 3447-3452, vol. 4.

[8] —- "Lyapunov based adaptive control of a mems gyroscope," in Proc. 2002 American Control Conf., 2002, vol. 5, pp. 3765-3770.

[9] M. Lemkin and B. Boser, "A three-axis micromachined accelerometer with a cmos position-sense interface and digital offset-trim electronics," IEEE J. Solid-State Circuits, vol. 34, no. 4, pp. 456-468, Apr. 1999.

[10] H. Luinge, "Inertial Sensing of Human Movement" Ph.D. thesis, Twente Univ. Press, Twente, The Netherlands, 2002 [Online]. Available: http://www.tup.utwente.nl

[11] C. W. Mace and Owen, Gpsi Dead Reckoning White Paper: Product Overview Neve Technologies, White Paper, Feb. 2001.

[12] R. M'Closkey, S. Gibson, and J. Hui, "System identification of a mems gyroscope," Trans. ASME. J. Dynam. Syst., Measur., Contr., vol. 123, no. 2, pp. 201-210, 2001.

[13] C. Painter and A. Shkel, "Active structural error suppression in mems vibratory rate integrating gyroscopes," IEEE Sensors J., vol. 3, no. 5, pp. 595-606, May 2003.

[14] S. Park, "Adaptive Control Strategies for Mems Gyroscopes," Ph.D. dissertation, Univ. California, Berkeley, CA, 2000

[15] S. Park, R. Horowitz, and T. Chin-Woo, "Adaptive controller design of mems gyroscopes," in Proc. IEEE Intelligent Transportation Systems, 2001, pp. 496-501.

[16] S. Sastry, Adaptive Control Stability, Convergence, and Robustness, ser. Prentice Hall Information and System Sciences Series. Englewood Cliffs, NJ: Prentice-Hall, 1989.

[17] A. Shkel, R. Horowitz, A. Seshia, S. Park, and R. Howe, "Dynamics and control of micromachined gyroscopes," in Proc. American Control Conf., 1999, vol. 3, pp. 2119-2124, vol. 3 .

[18] T. Singh and T. Ytterdal, "A single-ended to differential capacitive sensor interface circuit designed in CMOS technology," in Proc. 2004 Int. Symp. Circuits and Systems, 2004, vol. 1, pp. I-948-I-951, vol. 1.

[19] N. Yazdi, F. Ayazi, and K. Najafi, "Micromachined inertial sensors," Proc. IEEE, vol. 86, no. 8, pp. 1640-1659, Aug. 1998.

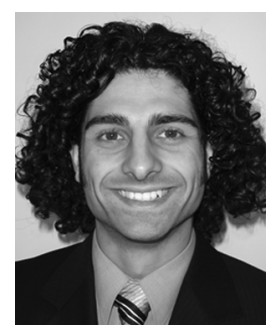

James D. John (M'03) received the B. Eng. degree (Hons.) in the field of mechatronics in 2000 from Deakin University, Australia, where he was awarded the Sir Alfred Deakin Medal. He is currently pursuing the Ph.D. degree in the area of micro electro mechanical systems (MEMS) at RMIT University, Melbourne, Australia, focusing on angular rate sensing.

He was previously with Motorola, Adelaide, Australia, in their software development center.

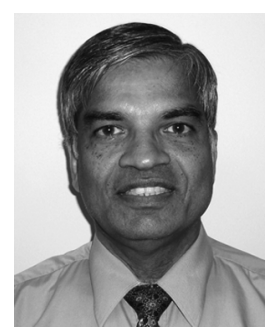

Thurai Vinay (M'02) received the B.Sc. Eng. degree from the University of Ceylon, Sri Lanka, in 1968 and the $\mathrm{Ph} . \mathrm{D}$. degree from the University of Birmingham, Birmingham, U.K., in 1974.

$\mathrm{He}$ is a Senior Lecturer in the School of Electrical and Computer Engineering, RMIT University, Melbourne, Australia. He was a Researcher and Project Leader in the CRC for Microtechnology, Australia, during 2001-2004. His research interests are in control systems, mechatronics, and micro electro mechanical systems (MEMS). 